\title{
Survey on Energy Efficiency in Cloud Computing
}

\author{
Lakshmi Digra $^{1}$ and Sharanjeet Singh ${ }^{2}$ \\ ${ }^{1}$ PG Student, ${ }^{2}$ Assistant Professor \\ Guru Nanak Dev University, Gurdaspur, Punjab, India \\ E-Mail: lakshmidigra7@gmail.com, sharan_sran@yahoo.co.in
}

\begin{abstract}
Data centers are serious, energy-hungry infrastructures that can run large scale Internet based services. Energy ingesting representations are essential in designing and improving energy-efficient operations to reduce excessive energy consumption in data centers. This paper presents a survey on Energy efficiency in data centers, importance of energy efficiency. It also describes the increasing demands for data center in worldwide and the reasons for data centers energy inefficient? In this paper we define the challenges for implementing changes in data centers and explain why and how the energy requirements of data centers are growing. After that we compare the German data center market at international level and we see the energy consumption of data centers and servers in Germany from 2010 -2016.
\end{abstract}

Keywords: Survey, Energy Efficiency, Cloud Computing

\section{INTRODUCTION}

The meaning of energy efficiency is using less energy to render the similar facility. There are many benefits to using less energy in our households. Energy efficiency helps our environment from the carbon dioxide emission. In its position of introducing natural gas and electricity from outside of your community, national and local companies can provide energy efficient facilities and undertake. At its most basic, energy efficiency denotes to a technique of sinking energy consumption by using less energy to make the same mass of beneficial output. For instance, an energyefficient 12W LED bulb uses $75-80 \%$ less energy than a $60 \mathrm{~W}$ out dated bulb but gives up the same level of brightness.

\section{A. Energy Efficiency in Data Center}

In any data center, the benefits of an energy efficient project and act of structure and organizations for both the facility and IT will cut prices (both CapEx and OpEx), and help administrations follow to industry energy standards and government regulations - adding a competitive edge to the line. When smart energy management is added into the equation through many strategies such as right-sizing tackle, enhancing the atmosphere and proper prior planning, all data centers, cloud co-location and multi-tenant workers can have a formula for a successful, profitable and highly efficient presentation and profession.

\section{B. Benefits of Energy Efficiency}

1. People use $85-90 \%$ of their time inside - at work, college and every day. An efficient house sustains sensible temperatures, little humidity, increased air quality and energy efficient houses use less energy and charge less to activate and yield fewer greenhouse gasses, which is harmless for you and the surroundings.

2. Refining your house or workshop's energy efficiency will save you money. For instance, the normal owner contributing in the Live Smart BC grant program will reduce energy operation by about 30 percent, proposing financial investments of $\$ 600$ on a $\$ 2000$ once a year heating bill.

3. By energy efficiency retrofits, the usual household made 25 years ago has the potential to take down their energy bills by $40 \%$ and to functional the amount of greenhouse airs the house produces by 5 tons per year. Working down the full amount of greenhouse gasses in the air is an important fact of sensation the peculiar effects of temperature alteration, both close and widespread.

4. Energy efficient buildings usually have extended life cycles, lesser repairs charges, and cost less to run. Rental properties are easier to live in, are rapidly occupied, and report lower tenant turnover rates. The natural light, calm temperatures and increased inside air quality of energy efficient commercial building results in increased staff production. And homeowners advantage from improved control over their energy bills while being in a place that cares their life style demands.

\section{Energy Efficiency and Other Green Housing Techniques Helping the Atmosphere}

Decreasing your energy use can reduce the amount of energy your appliance needs to produce, which you can successfully reduce your little greenhouse gas productions (and your carbon foot-mark) with energy efficiency procedures. In the enumeration of the direct economic and ecological benefits of consuming energy efficiency methods, mixtures of energy efficiency with other "green" practices can outspread even more win.

\section{INCREASING DEMANDS FOR DATA CENTERS}

In today's quickly increasing digital economy, customer request for more data and high-bandwidth contented is stimulating the surge in data center occupational. While it comes as no amazement there is an insatiable need for other computing power (given the attractiveness of Facebook, 
Instagram, email, and the Internet of Things), few regulars realize just how dynamically exclusive our "green" electronics are. In a 2013 boom since the Digital Power Group (DPG), CEO determines the typical iPhone ingests additional vigor than a medium-sized freezer from the Biological Safety Activity's Vitality Star ratings list 362 $\mathrm{kWh}$ of authority per year for iPhone wireless systems, material procedure and portable alleging versus $322 \mathrm{kWh}$ per year for a deep-freeze.[1] While the quantity of dynamism used by several android phone will fluctuate extensively based on user procedure, this exposure authorizes a serious energy anxiety allowing for the current predictable amount of android phone users will exceed 2 billion in 2016.[2] To embrace all this ordinal action, added than 1.8 trillion gigabytes of numerical files were made universally in 2012, adding over 3 million data centers.[3] Assumed the path of the ordinal economy, these records are only predictable to increase, with no goal in sight for sustaining the mandate for supplementary material.

\section{A. Reasons for Data Center Energy Inefficient}

When it comes to data centers, a slew of likely distrusts contribute to energy inefficiency, including over-sizing power and cooling schemes, poor capacity planning, underutilized IT apparatus, colder than needed space temperatures and stranding capacities (power, cooling, and blank). Handling and adjusting the data center process, size (power, space, refrigerating) in a composite, fast paced and changing environment of a day to day basis are stimulating, mainly when it comes to Cloud, Wholesale and MultiTenant Collocation suppliers.

One of the main reasons data centers has such high energy inefficiency is largely a significance of the mutual correlation between users demanding extra processing power and the businesses that risk trailing business if they disregard to fulfill consumer need. As a significance of this, most approaches run their data centers at full capacity 24/7 and to guard beside power failures, they will install supplementary stores of generators that produce diesel exhaust. The fear of data center failure results in enormous underutilization of the tools for the majority of the time, since the structures are way over designed for the worst case picture. The NRDC cites the usual server functions, at no more than 12 to $18 \%$ of its extreme capacity, however still drawing 30 to $60 \%$ of its supreme power.

Several new major source of inefficiency comes from bad practice and accepted up to confirm to the latest knowledge. NRDC, in organization with An thesis, reports that up to 30 $\%$ of servers are "comatose", or obsolete and no longer needed, but still plugged in and overwhelming electricity around the clock, while other equipment are grossly underutilized.

In a curious statistic by NRDC, the most important offenders of data center inefficiency are not the large organizations classically connected with high presentation computing, but less visible, low and medium sized data centers, which lag behind in efficiency and assumption of different technology. In fact, hyper-scale cloud computing data centers only make up $1 \%$ of data center energy ingesting in the United States, while small and medium sized data centers are responsible for $49 \%$ of U.S. server power usage.

\section{B. Challenges for Implementing Change}

The most important cause of data center inefficiency poses numerous obstacles toward delivering energy. One worthy word by the NRDC is to contract out computing procedures using the multitenant data center specialized model. [4] Inappropriately, there are consistently several experiments related with bringing this good instance. Multi-tenant data center operators have little incentive to offer an energyeffective facility to their customers, since their primary objective is to hold charges down and reserve high levels of sanctuary, trustworthiness, and uptime. [6]

The extra time and money to set up and display apparatus for more beneficial energy efficiency is of lower priority compared to associating space and power capacity to reserve for more consumers in the current commercial model. [4] Likewise, consumers of multi-tenant data centers commonly have separate sections for those paying the data center power bill and the IT section managing data centers Operations. Only $20 \%$ of IT sections handle the power bill, a statistic that has remained motionless in over 5 years. [4]

\section{GROWTH OF THE ENERGY REQUIREMENTS OF DATA CENTERS}

The energy efficiency of information centers has improved speedily above the age. In the times of the abundant data center boom in the 2000s, the energy efficiency of information centers has been only just a matter of curiosity. Simply in excess of the period was the accumulative power consumption of the combined amount of IT apparatus in businesses predictable. IT may previously have simply been a negligible power consumer like the light, but the supercomputers became a chief source of power use of goods and services in many pots. The power consumption of data centers improved by $150 \%$ between 2000 and 2008 . Only at that time, most businesses weren't even awake of the quantity of power their IT was consuming. 10 years ago, not even $10 \%$ of data center operators were able to present data on the power requirements of their data centers.

\section{A. The Efficiency of the Computing Power is Increasing Rapidly - The Number of Calculations Per KWh Doubles Every 1.5 Years}

This is moderately changed nowadays. Indeed, nearby are still data centers commonly negligible ones wherever power consumption is scarcely observed and these frequently resolves actual incompetently. Only for the irresistible essence of data centers and all huge operators' inordinate 
significance is placed on energy efficiency today. Data center operatives are reinforced in this over and completed with always better and more operative information. The effectiveness of the computing power is growing speedily the number of calculations per $\mathrm{kWh}$ doubles each 1.5 years. Computing power can be provisioned extra adaptably and professionally today through virtualization and cloud computing. The data center basis substructure is similarly improving constantly. Uninterruptible power supply systems (UPS) today attain almost $100 \%$ efficiency. 10 years ago, the freezing of a data center frequently amounted to $50 \%$ of the power consumption, where today this amount is extra like $20 \%$ or equal fewer.

\section{B. The Power Consumption of Data Centers in Germany Grew Strongly again in 2016, By $4.2 \%$ to 12.4 Billion Kwh.}

No, quite the opposite. The power consumption of data center in Germany raised stalwartly yet again in 2016, by $4.2 \%$ to 12.4 billion $\mathrm{kWh}$. This means data centers in Germany want as much power as 4 medium-sized coal-fired power stations create in a year. Presently, the power necessities are essentially increasing more powerfully than in previous years despite the achievements in the area of efficiency. The reason for this is above all the strong development of data center abilities in Germany. Many new data centers are being built almost every week the press report of additional new large data center is released.

\section{Causes of the New Bang in Data Centers in Germany}

The growing digitization of production and civilization is one important source. More and more devices and sensors are actuality linked to the Internet experts guess that by 2020 this will be around 50 billion devices. Worldwide, there are already about 5 billion mobile phones and tablets and a central processing capacity is mandatory for the role of most apps. The new data capacities produced every day are huge. One example: 4 billion images are shared every day on social media. Presence in Germany, one more problem is also essential to the data center development: the considerations on the topic of data safety and information safeguard. It is a must for three stations of German companies that the data centers where their cloud services are hosted are situated in Germany. Many American Internet hulks are therefore spreading their own data center capacities in Germany, in mandate to satisfy the needs of their clients here.

\section{German Data Center Market in International Comparison}

Globally, energy use of goods and services is growing considerably faster than in Germany between 2010 and 2015 alone; the power expenditure of data centers worldwide grew by $30 \%$. This means that the worldwide energy consumption grew at double the speed of the German demands. There are two primary reasons why energy consumption grew less strongly in Germany.
Initially, the German data centers counts among the most energy efficient data centers in the world.

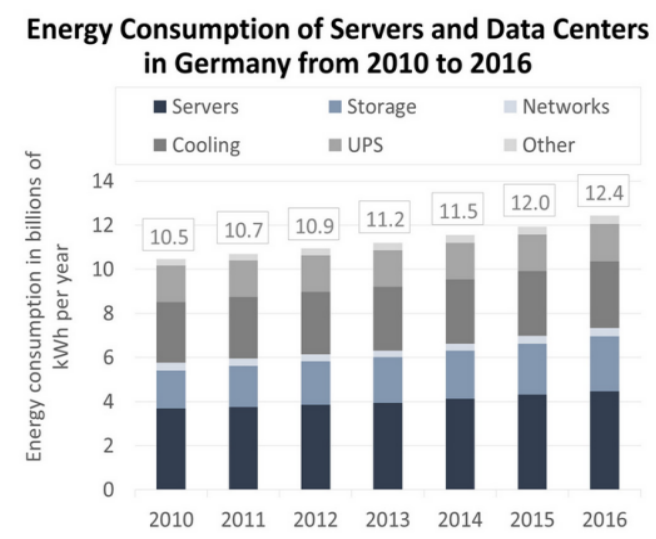

Fig.1 Energy Consumption of Servers and Data Centers

The German shop is a leading market place for energy efficient data centers. This is the case on the one hand for data center operators who are, in global valuations, very complex to the energy use of their data centers. But it's similarly the case for data center contractors. Many energy efficient data center machineries come from Germany.

The another cause for the relatively moderate progress of the energy usage of German data centers is that, in spite of the present boom, advancement in the German data center shop is average in the midterm. Grocery stores in Asia, North and South America, and also in Scandinavia are growing significantly more solid. The details for this comprise the moderately great power charges and the comparatively time-consuming permitting procedures of data centers in Germany. The abundant significance of data centers for the ordinal economy of the future has barely put down the responsiveness of German policy manufacturers up to today. Several other states encourage the Greenfield expansion of data centers much more mightily.

In Sweden and Finland, for instance, there are tax reductions for big data centers. If Germany is to take a defining role in digitization as a striking data center location, it will include a rearrangement of political ideas in a number of posts here.

\section{STEPS FOR IMPROVEMENT}

One suggestion for development is virtualization (the act of consolidating multiple servers to a single physical server), which can allow for more efficient capacity use and cut energy prices. Watching from the issues discussed in "Why Data Centers are Inefficient", organizations can also cut down the number of comatose servers, expand storage utilization with more serious organization of data, invest in more energy-efficient server technologies, and manage hot/cold air temperature flow in data centers. Correspondingly, on the lines of implementing newer expertise and energy-effective apparatus, major technology 
companies such as APPLE and GOOGLE are using renewable energy fonts to decrease carbon releases and rearranging power-hungry data centers nearby unswerving causes of renewable energy. [1] In recent years, the data center business has approved modest server exploitation metric to systematize and estimate the power use effectiveness (PUE) of data centers. Since most data centers use almost as much non-compete or "overhead" energy (i.e. cooling and power conversion) as the hosts themselves, PUE has been defined as the ratio between total facility energy and IT apparatus energy.[7] The acceptance of the PUE provides visibility of the efficiencies of data centers and creates incentives to optimize for this metric.

Although there are many ways to cut the energy use of goods and services within an administration's data center, the larger problem, however lies with the misalignment of inducements between the data center operators, service providers, and multi-tenant consumers. To ease this issue, NRDC further recommends multi-tenant data center stakeholders to produce a "green tenancy" contract template to incentivize energy reserves and public disclosure of data center energy and carbon performance.[4] Of course, there are still many hurdles with substantial organizations to do either of these, as remarked in "Challenges for Implementing Change", but even a $40 \%$ reduction in energy utilization, which is just half of the precisely possible reduction, according to the NRDC, would generate $\$ 3.8$ billion in savings for productions [4].

\section{CONCLUSION}

In this paper, we emphasized the energy efficiency in data centers, reasons for data centers inefficient and steps for improvement. It is expected that this survey will be highly useful for further research on how we use the different techniques for reducing the energy efficiency in data center the solution is green computing. In future work we will define the different techniques for energy efficiency in data centers like provisioning, consolidation, virtualization etc.

\section{REFERENCES}

[1] B. Walsh, "The Surprisingly Large Energy Footprint of the Digital Economy", Time, 14 Aug 2013.

[2] W. Curtis, "Quarter of the World Will Be Using Smartphones in 2016", The Telegraph, 11 Dec 2014.

[3] J. Glanz, "Power Pollution and the Internet", New York Times, 22 Sept. 2012.

[4] P. Delforge, "America's Data Centers Are Wasting Huge Amounts of Energy", National Resources Defense Council, Issue Brief 14 -08- A, August 2014.

[5] J. Lee, "Energy Usage of Server Farms", Physics 240, Stand Ford University, Fall 2012.

[6] G. Cullen et al., "Evaluation, Verification, and Measurement Study FY 2008/2009 Program for Silicon Valley Power", Summit Blue Consulting, December 2009.

[7] G. A. Brady et al. "A Case Study and Critical Assessment in Calculating Power Usage Effectiveness for a Data Center", Energy Convers. Manage, Vol. 76, No. 156, 2013. 